\title{
Rhythm and timing in chat room interaction
}

Book or Report Section

Accepted Version

Jones, R. H. (2013) Rhythm and timing in chat room interaction. In: Herring, S., Stein, D. and Virtanen, T. (eds.) The pragmatics of computer mediated communication. De Gruyter, Berlin, pp. 489-514. ISBN 9783110214468 Available at http://centaur.reading.ac.uk/66522/

It is advisable to refer to the publisher's version if you intend to cite from the work. See Guidance on citing.

Publisher: De Gruyter

All outputs in CentAUR are protected by Intellectual Property Rights law, including copyright law. Copyright and IPR is retained by the creators or other copyright holders. Terms and conditions for use of this material are defined in the End User Agreement. 


\section{CentAUR}

Central Archive at the University of Reading

Reading's research outputs online 
020_Jones.pod 479

06-04-03 10:38:00 -mlb- mlb

IV. Discourse Pragmatics of computer-mediated communication Interaction 
020_Jones.pod 480

06-04-03 10:38:00 -mlb- mlb 


\title{
20. Rhythm and timing in chat room interaction Evidence from a gay chat room
}

\author{
Rodney H. Jones
}

\section{Introduction}

Considerable attention has been paid in pragmatics and related approaches to discourse, such as interactional sociolinguistics, conversation analysis, and mediated discourse analysis, to the role of rhythm and timing in voice-based communication. In face-to-face interactions and telephone conversation, for example, people have been found to respond rhythmically to one another in a continuous manner and are able to perform conversational transactions synchronously through a shared understanding of timing and transitional signals. Rhythm and timing are realized paralinguistically through pausing, pacing, and the use of stress and intonation (Couper-Kuhlen and Selting 1996; Erickson 1980) and in face-to-face communication, kinestically, for example through head movement, gesture, and gaze (Goodwin 2002; Kendon 1974).

Rhythm and timing have been seen to have especially important functions in the management of turns in conversation. Couper-Kuhlen (1993), for example, found in a large corpus of British and American data that speech rhythm serves as a metric for the timing of turn transitions. Goodwin (2002) has shown how prosodic aspects of speech are used in concert with gesture and posture to create multiple concurrently relevant temporalities within which participants interactively organize their interaction. Tannen (1984a, 1989) has observed how rhythmicity and repetition in talk aid in creating coherence in conversations and "involvement" between participants. Pragmatic studies in cross-cultural communication have shown that differences among speakers in behaviors such as speech speed, customary timing of turn-transitional pauses, and timing between periods of talk and periods of silence can lead to disfluencies and misunderstandings (Hall 1959; Lehtonen and Sajavaara 1985; Tannen 1984b).

Rhythm and timing are essential elements, not just in the organization and coherence of communication that occurs over time (van Leeuwen 2005), but also in the realization of speech functions and the negotiation of social identities and social relationships. Studies in interactional sociolinguistics have shown how timing and rhythm act as contextualization cues, framing utterances in particular ways (Gumperz 1982), and how they function in meta and phatic communication, providing information about speakers' attitudes towards their topics and their interlocutors (Tannen 1984a). Timing, rhythm, speed of speech, pausing, stress, and intonation are central ingredients in what Tannen (1984a) has called "conversational style". 
The role of rhythm and timing in computer-mediated communication (CMC) in particular, in text-based chat - has received less attention. The main reasons for this have chiefly to do with constraints of the medium. First, because of the reduced cues that characterize text based CMC, especially the muting of embodied modes like gaze and gesture, many ways of managing rhythm and timing used in "real-life" conversations are simply not available. These visual cues are responsible for most of the direct and immediate feedback about rhythm people get in face-to-face conversation. With it they regulate their interaction and synchronize with each other, making constant mutual adjustments based on timing (Mantovani 2001).

Second, even many of the verbal cues we use to organize pacing and turn-taking in our face-to-face conversations cannot be exploited in computer chat. In most popular chat programs, including the one I am concerned with here, information about real time turn development is not revealed - turns are only transmitted when users press the enter key. Garcia and Jacobs $(1998,1999)$ call chat "quasi-synchronous communication", for while there is a semblance of synchronicity, because turns are constructed in isolation from other participants and only become accessible once posted, there is not the same immediate and mutual access to utterances-in-production that is characteristic of face-to-face interaction. With the process of message production separated as it is from message transmission, choices in turn negotiation are severely limited. Interruptions in the conventional sense, for example, are impossible (Herring 1999). The meaning of pauses is also problematic, because a sender has no access to the recipient's actions until a reply is posted (Rintel, Pittam, and Mulholland 2003). Extraneous contextual factors such as typing speed, the speed of the connection, possible technical problems, and the fact that most chats are part of multiple concurrent activities (Jones 2003) also make the analysis of the communicative uses of rhythm and timing in chat more difficult and less reliable.

A particularly important reason for the lack of research on timing in chat is the fact that most of the data collected in studies of CMC are not really suitable for analyzing the complex temporal features of multiparty chat (Bays 1998). Most data consist of text based "histories" or logs of chats which do not take into account the additional chatting, email, instant messaging, and other communicative activity that may have been occurring co-terminus with the messages under analysis. Although these transcripts are sometimes marked with the time messages appeared on the server, this is often not a reliable indicator of the timing actually experienced by users at their screens.

Because of these limitations, less attention has been paid to the strategies users have developed around rhythm and timing within these constraints, and more has been paid to designing systems to make the rhythm and timing of computer chat more like that of face-to-face interaction. Many chat and instant messaging clients (like MSN Messenger) now have awareness indicators that show when another 
person is typing, and other innovations are being experimented with. Shankar and her colleagues (2000), for example, propose a way of representing multiparty chat that is inspired by musical scores. However, such attempts have not become widely available, and it is questionable how popular they would be if they were.

This chapter argues that users of computer chat have developed ways to exploit timing and rhythm for a multitude of communicative purposes within even the most minimalist framework for mutual monitoring. Text-based CMC, as BenZe'ev (2004) points out, is both lean and rich: lean in the reduced cues it makes available and rich in the ways participants use these cues to create the strong sense of intimacy and solidarity associated with what Walther (1996) calls "hyperpersonal communication". Numerous examinations of computer chat have noted the importance of timing and rhythm in turn taking and turn construction (see for example Bays 1998; de Siqueira and Herring 2009; Markman 2004; Rintel and Pittman 1997), as well as in the establishment of particular levels of formality in interaction and the development of feelings of closeness or "presence" between interlocutors (Ben Ze'ev 2004; Donath 2004). Bays (1998), for example, argues that the desire to maintain a particular rhythm in relation to their interlocutors is a factor affecting the linguistic construction of turns by users of IRC (Internet Relay Chat), and de Siqueira and Herring (2009) found that participants in IM (Instant Messaging) sessions tend to vary the timing of their responses to harmonize with their interlocutors.

Timing has been found to be important even in asynchronous forms of CMC such as email. Walther and Tidwell (1995), for example, argue that email communication often depends on "chronemics" or "time-related messages" to convey nonverbal cues, and claim that time is an "intrinsic part" of this type of social interaction conveying meaning and defining the nature and quality of relationships with others "across multiple levels" (361). Similarly, Kalman et al. (2006), in their examination of response latencies in asynchronous $\mathrm{CMC}$, noted that response time occurred with mathematical regularity with most responses created quickly and delays in responding resulting in fewer subsequent responses.

In fact, it can be argued that time is even more important in CMC, precisely because of some of the reasons mentioned above - the "narrow bandwidth" and reduced cues of computer chat - signals such as language, style, timing, and speed of writing become particularly salient. According to Lea and Spears (1995: 217), "even first-time users form impressions of other communicants' dispositions and personalities based on their communication style", which, in part, involves response time, rhythm, typing speed, and other temporal factors.

This chapter illustrates these issues with a study of the pragmatic uses of rhythm and timing in interaction among participants in a Hong Kong based gay chat room. It seeks to show how timing is used as a semiotic resource in the accomplishment of particular concrete social actions within the larger social practice of "fishing" (searching for sexual partners). The data were collected as part of a 
six-month long ethnographic study of this chat room which involved hiring 20 regular users as "participant-researchers" to help in setting goals and plans for the research, interviewing, and securing consent from their chat partners and monitoring their own computer use. Participant-researchers also took "screen movies" of their interactions in the chat room, capturing the temporal aspects of their multiple concurrent interactions with other users.

The chat room itself uses a Java-based chat client, which allows users to enter a room with a username and profile that can be changed every time one logs in. Upon entering, one's username appears on the list of other current users of the room. Clicking on a particular name opens a private chat window with that user. Users can also take part in public chat though the main console, but users rarely do so, preferring to go directly to private conversations with other occupants of the room. This particular chat client gives no indication of turns in progress. Participants' contributions are sent only after the user presses "enter" and are received complete on their interlocutor's computer screen within seconds, depending on the speed of the connection. Users frequently carry on chats with multiple users, or attend to other tasks like checking email or looking at pornography while engaged in chatting.

The data were collected using a software program called Spector, which participant-researchers installed on their computers. The program took screen shots of users' computers at fixed intervals of three seconds. The advantage of this kind of data is, first, that it allows the analyst to experience rhythm and timing in a way similar to that experienced by the user, and second, that it gives the analyst access to all of the different activities that might be occupying the user's time rather than relying on individual chat logs isolated from their context. Although taking screenshots at three second intervals (the setting determined to least interfere with the speed of the users' computers) limits the precision of our analysis when it comes to timing, because the time between turns tends to be much longer in chat than in face-to-face communication (see below), this range of data can at least reveal more general patterns of timing and rhythm that exist in this chat room and suggest areas where later work can focus.

Different media amplify and constrain users' orientations towards time and space in different ways, making some ways of interacting and making meaning more possible, and others less possible (van Leeuwen 2005). At the same time, different activities also presuppose particular orientations towards time and space, what I have previously referred to as "attention structures" (Jones 2005); different practices have different rhythms. My concern in this chapter is to show, given the constraints of this medium, how participants cooperate to produce "shared times" and "shared rhythms" within which this particular social practice is framed, how they develop conventions about the meaning of different time related aspects of their interaction, and how timing is used to create synchrony and feelings of intimacy. 
In the particular environment I examined, used primarily for meeting people for quick sex, rhythm and timing play a crucial role in whether or not one is successful in this social practice. Timing is important in the creation of interactional involvement and the discursive construction of desire. It plays a role in the power users exercise over each other and, in the kinds of mutual pleasure that they share. The "literate" practice of searching for sexual partners in this chat room requires understanding not just what individual messages mean but also the proper procedures with which they are to be delivered within the chronological framework of the activity.

\section{Timing and attention}

One of the most important features of the interaction investigated here is that it is extremely instrumental, i.e. directed towards the goal of sexual contact. Interactants are sometimes quite efficient in their pursuit of this goal: When asked how long the interval between making on-line contact with a potential partner and finalizing plans for actually meeting up for sex might be, one participant said, "very short ... perhaps as short as few minutes". The second important feature of this kind of interaction is that it is intensely competitive. In the "marketplace" (ten Have 2000) of the chat room, users constantly compete for "interactional time" with other users who might want to engage the same target, and, at the same time, they distribute their own time and attentional resources among a number of different targets.

This marketplace, then, operates according to a strict "attention economy" (Lankshear and Knobel 2002) where the most valuable asset is the attention of others, and the investment one makes to gain it is paying attention back to them. The issue of attention goes much deeper here than sexual attraction. Back in 1951, Reusch and Bateson wrote that the foundation of all social situations is some form of mutual perception. The form this mutual perception takes determines how we enact social presence, display our attentional state, and frame the kinds of activities we are involved in. In face-to-face conversation, both speaker and hearer must actively respond to what transpires by signalling involvement, either directly through words or indirectly through gestures or similar nonverbal signals like gaze, posture, and bodily alignment (Tannen 1989). However, in CMC, in which non-verbal bodily cues are not available, the "state of talk" becomes more tenuous; only when one's message is visible in the scrolling text window does one truly "exist" in the conversation. Therefore, the only way users have to be present to their interlocutors and communicate attention or involvement is by replying to them within a particular period of time. Similarly, though interpreting their timing (in particular the time between turns) users make judgements about their social presence, social activity, and attentional state. 
Timing therefore has an important interpersonal function in this type of interaction, the length of pauses between turns usually showing involvement and interest or "seriousness" about continuing the interaction. Shorter pauses often show a higher degree of interest and attention, while longer pauses usually communicate a lack of interest, or even a lack of "presence" in the conversation, and often result in interactions being terminated. Of course, as I will show later, what counts as "short" and "long" can vary considerably across contexts, and even across different stages of a single conversation, with pause length taking on different pragmatic functions.

The importance of the length of pauses between turns is testified to by how sensitive users themselves are to it. One participant stated: "long delays without response are generally interpreted as 'he is ignoring me ...'." Another said:

I basically cannot tolerate any silence for more than 2-3 minutes. Those who fail to respond within 3 minutes, I will assume that they are not interested in carrying on the conversation. Those who respond promptly I will see that as a sign of their interest and good integrity. I also tend to use their response time to see how serious they are in making a conversation.

Here, the interpersonal function of timing extends to making judgements not just about one's interlocutor's attitude but also about his character ("integrity"). Time, however, is relative; how long "too long" is depends on a number of factors, including the personality and psychological state of the user and the desirability of his target. As one participant put it:

If I am really horny at that moment, I would say reply within 10 seconds is acceptable. if more than that, I would guess the guy is no longer interested in me, unless he told me to wait. If I am not truly horny, just want to fool around in the chat room, replying within 15 to 20 secs is acceptable.

Another stated:

It depends on my mood ... but I also try to imagine what might be happening at the

other end, like multiple chat, organizing the desktop, might be away from the computer. Also try to factor in how long it $\longrightarrow$ ld take me to respond etc. To put a time on it ... maybe a minimum of 5 secc through to minutes but no longer than 3 minutes.

As can be seen in these statements, what is considered an acceptable length of a pause between turns is highly variable, ranging from 10 seconds to three minutes. This relativity in time can lead to misunderstandings when two users do not share the same sets of expectations, or when delays that result from typing difficulty or the other user momentarily leaving his computer are misinterpreted as rejection. Garcia and Jacobs (1998) in their analysis of timing and sequencing distortions in chat found that a significant portion of chat turns were used to clear up confusion caused by prior turns (see also Smith and Burkhalter 2000). In the activity type I am 
considering, however, such repairs are not as common, since misunderstandings almost always end with interactions being terminated. This means that the development of interactional synchrony (see below) fairly early on in these encounters is particularly important.

One thing that must be remembered when we think about how timing is used to maintain conversational involvement is the lag time in CMC between when utterances are initiated and when they are actually received. According to Walther (1996), it takes four to five times longer to exchange CMC message than to exchange messages in face-to-face conversation. Users adapt to the peculiarities of turn taking that the media imposes by being sensitive to the "lag time", understanding, for instance, that, if two turns appear within one second of each other, it is probably clear that the second turn was not intended to be a reply to the first (Herring 1999).

Faced with the demands of "being present" for one's interlocutor (perhaps several interlocutors) and sustaining the interaction, participants tended to employ the strategy of making their turns short, allowing for more rapid exchanges. Most turns in my data are from one to four words long. When participants have something longer to say, they usually break it up across several consecutive turns. The rapid back and forth that characterizes (successful) chats often also involves the use of filler terms like "o" and "ic" to mark users' rhythmic place in the conversation at times when they cannot think of anything to say or they wish to shift the onus to the other user to introduce the next topic. These short turns are a common characteristic of "multitasking" in which people manage diverse demands on their time and attention with brief and irregular responses (Jacobson 1999). The length of contributions in such situations is restricted by what Millard (1997: 159) calls "chrono-economic stress", defined in terms of the user's awareness of the limits to the communicative resources (time, attention, bandwidth) that can be devoted to any given exchange.

A typical conversation can be seen in example 1, in which each of the table signifies the passing of three seconds, the intervals at which the software was set to take screenshots, and in which the unnamed user (indicated with >) is the user from whose computer the screenshots were taken.

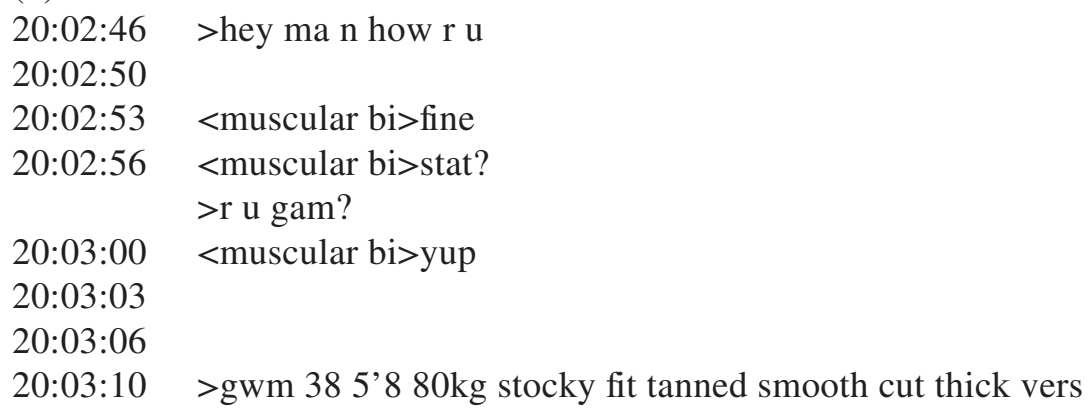




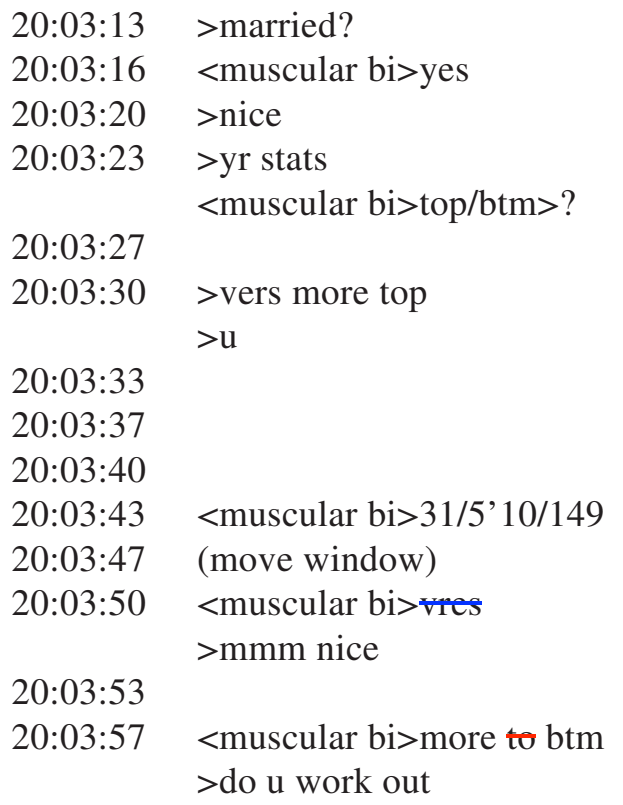

As can be seen, participants take short, rapid turns with pauses between turns usually no longer than six seconds. Longer pauses do occur notably after participants are asked for their "stats" (self description involving age, weight and height, and sometimes other relevant information about physical appearance), primarily because these responses tend to take a bit longer to type, especially if they are elaborated upon (as in: "gwm 38 5' 8 stocky fit tanned smooth cut thick vers"1). Most turns consist of one to four words. The string of descriptors: "gwm 385 , 8 stocky fit tanned smooth cut thick vers" is unusually long, not only creating a slight interruption in the rhythm of the conversation but also creating implicature, communicating particular involvement, cooperation, and interest.

As the interaction progresses, however, this rhythmic pattern begins to break down. At 20:30:23, a slight disfluency occurs when both parties ask a question of each other at the same time. Such an occurrence in itself is not particularly problematic - users are used to them, and the initiator of the chat immediately compensates by changing his discourse position (from questioner to answerer) and answering muscular bi's question before muscular bi finally answers his. What is notable, though, is that while the first response comes within six seconds of the question, the response from muscular bi comes more than fifteen seconds after the question.

Finally, muscular bi does not respond to the question "do you workout" (example 2). After waiting around 20 seconds, his interlocutor makes another attempt to elicit a response by asking a different question, "any pic?", after which he waits another 33 seconds or so before issuing a more explicit prompt, "hello", and then after approximately 30 more seconds finally gives up. 
(2)

20:04:00

20:04:04

20:04:07

20:04:10

20:04:14

20:04:17

20:04:20

20:04:24

20:04:27

20:04:31

20:04:34

20:04:37

20:04:41

20:04:44

20:04:47

20:04:51

20:04:54

20:04:58

>hello

20:05:01

20:05:04

20:05:08

20:05:11

20:05:14

20:05:18

20:05:21

20:05:24

20:05:28

20:05:31 (close window)

One might ask why muscular bi does not terminate the conversation himself if he is not interested. One can never be sure, however, if such pauses indicate a lack of interest or are due to other extraneous circumstances (the user may be answering a phone call or attending to chats with other - perhaps more desirable - partners). At the same time, this strategy of showing lack of interest is a particularly prevalent means of rejection. The preference for this means of termination, rather than the less popular "sorry", "gotta go", or "not my type", comes from the fundamental difficulty involved in "ending" characteristic of all communication (Schegloff and Sacks 1973), magnified by the even more "face threatening" nature of termination of this particular kind of interaction. In the entire corpus of chats collected, $86 \%$ are terminated without any closing ritual. 
At the same time, very short pauses can communicate intense interest. Just as in face-to-face conversation, people have different "conversational styles" (Tannen 1984a), and one aspect of style has to do with how much (and how) one shows involvement. Showing involvement in computer chat as in face-to-face conversation often includes talking a lot and taking shorter pauses between turns. Tannen (1984a) calls this "high involvement style", and, aside from shorter pauses, it also includes features such as more frequent questions and, in face-to-face conversation, paralinguistic, proxemic, and gestural characteristics.

As I have already mentioned, most conversations in this chat room seem to be characterized by "high involvement", when they are progressing successfully that is; interactions not showing such involvement usually do not last. There are instances, however, in which this involvement is even more pronounced. The main feature of this "hyper-involvement" style is when a user issues a rapid series of consecutive turns without waiting for a reply, as in example 3.

(3)

09:20:26<LeanFit>nice stats

09:20:30 <LeanFit>i like

09:20:34 <LeanFit>i am lean fit here

09:20:38 >ic

$<$ LeanFit $>$ looking for?

09:20:42 >any

09:20:46 <LeanFit>I look for

09:20:50

09:20:55 <LeanFit>regular gay sex buddy

09:20:59 <LeanFit>ok to u?

09:21:03

09:21:07 <LeanFit>but

$>$ sure

$>$ but what

09:21:11 <LeanFit>we can swim together

09:21:15 >haha

$<$ LeamFit $>$ and have sex

09:21:19 <LeanFit $>$ ok to $u$ ?

09:21:23<LeanFit>hei

09:21:27 > why not

09:21:31<LeanFit>wanna try swim at night in beach with me?

09:21:36

09:21:40 >i wanna to go this afternoon

09:21:44 <LeanFit>sure

09:21:48

09:21:52<LeanFit>where shall we go? 
09:21:56 <LeanFit>pool?

09:22:00 <LeanFit> where u live?

In this example, LeanFit's contributions come at rate one every 5.7 seconds, and most intervals are three seconds or less. In the space of the one minute 26 seconds of this excerpt, LeanFit takes 15 turns while his interlocutor takes six. This strategy not only gives the user a way to highlight his attention towards his interlocutor, but also a way of keeping the interlocutor's attention by continually "refreshing" his presence on his interlocutor's screen. One's use of such a rhythmic strategy, as in face-to-face conversation, can affect others' perception of one's character; users who have different conversational styles might interpret such strategies as "friendly", or as "pushy" or "desperate" (Tannen 1984a).

This use of rapid, consecutive turns also has the function of maintaining presence by avoiding the longer pauses that might ensue when longer stretches of information need to be given, such as "stats". In example 4, for instance, hot fun divides his "stats" into five turns.

(4)

$\begin{array}{ll}20: 26: 11 & >\text { ur stats } \\ 20: 26: 15 & \\ 20: 26: 18 & <\text { hot fun> } 21 \\ 20: 26: 25 & <\text { hot fun> } 180 \mathrm{~cm} \\ 20: 26: 28 & <\text { hot fun> } 1501 \mathrm{bs} \\ 20: 26: 31 & <\text { hot fun> } 30 \mathrm{w} \\ 20: 26: 35 & <\text { hot fun> }>41 \mathrm{c}\end{array}$

This rhythmic incremental release of information, however, can also serve another function, that of introducing "suspense" into the process of seduction. Here, rhythm plays a crucial role in the game of revealing and withholding information to maximize involvement from one's interlocutor (van Leewuen 2005). Hot fun's incremental release of his statistics becomes a kind of textual striptease (Barthes 1975) that heightens desire by heightening anticipation of the next, partial revelation. A similar strategy is used by Naked Sleeper when he describes what he likes to do in bed (example 5).

(5)

$$
\begin{array}{ll}
20: 38: 37 & >\text { what do u like to do in sex? } \\
20: 38: 42 & \\
20: 38: 47 & \\
20: 38: 52 & <\text { Naked Sleeper }>\text { kissing } \\
20: 38: 57 & <\text { Naked Sleeper }>\text { oral } \\
20: 39: 02 & <\text { Naked Sleeper } \text { fucking }
\end{array}
$$




\section{Conversational management}

I do not mean to suggest that long pauses always show lack of interest or result in interactions being terminated. It depends very much on where these pauses occur in the conversation. Conversations are not mono rhythmic - they develop through many changes in rhythm and, in fact, as in face-to-face conversations, these changes of rhythm and shifts in tempo are often meaningful.

In fact, the average time between turns in my data is between 30-39 seconds, which does not seem consistent with the rapid turn taking we saw in the examples above. This is because these interactions tend to follow a pattern of "bursts" and "breaks" in which chunks of rapid interaction are interspersed with sometimes quite lengthy breaks.

Within these "bursts" of interaction the average time between turns is 3-9 seconds.

This segmentation of the conversation is by no means random. These bursts and breaks take place in the context of an interactional format which progresses through a fairly predictable set of stages made up primarily of question/answer adjacency pairs (the exchange of information about physical appearance, sharing of other information such as location and availability, the exchange of pictures, or the making of arrangements to meet). Each stage tends to take place in one burst, with breaks tending to occur between stages. The initial burst usually involves the exchange of information about physical appearance, the second stage usually involves further sharing of information (such as location and availability), the third involves the exchange of pictures, and the next involves arrangements to meet and often the exchange of telephone numbers. Breaks often serve to mark the transition to a new topic or new stage in the interaction. When they occur in between two halves of an adjacency pair or an offer of information and a reaction to it, they can create implicature that is interpreted sometimes as deliberation, sometimes as lack of interest, and sometimes as rudeness. Example 6 illustrates a typical pattern. In the first burst participants share information about what they are seeking and physical appearance.

(6)

$$
\begin{array}{ll}
20: 20: 23 & <\text { nice gam }>\text { hihi } \\
\text { 20:20:27 } & >\text { hi } \\
20: 20: 30 & <\text { nice gam }>\text { u lk for? } \\
\text { 20:20:33 } & \\
\text { 20:20:37 } & >\text { friends or fun } \\
20: 20: 40 & >\text { u? } \\
20: 20: 43 & <\text { nice gam }>\text { anything } \\
20: 20: 47 & >\text { great } \\
20: 20: 50 & <\text { nice gam }>\text { stats? }
\end{array}
$$


$20: 20: 53$

20:20:57

$20: 21: 00>36 / 6^{\prime} / 160$

20:21:03 <nice gam > nice stats

20:21:07 <nice gam> u gwm

20:21:10 > yup

20:21:13 > ur stats?

20:21:17

20:21:20 <nice gam> 21/5/11/130

20:21:24 > nice

Then after a break of about 20 seconds they talk about where they live (example 7). This exchange is followed by another break, also around 20 seconds long.

(7)

$20: 21: 27$

$20: 21: 34$

$20: 21: 37$

$20: 21: 40$

$20: 21: 44$

$20: 21: 47$

20:21:50

20:21:54 <nice gam> live alone

20:21:57 > yes

20:22:00 <nice gam $>$ where

20:22:04 > Tai Po

20:22:07 <nice gam> ic

20:22:11 <nice gam> quite close

$20: 22: 14$

20:22:17

20:22:21

20:22:24

$20: 22: 27$

After this the exchange of pictures is negotiated. The final break occurs after users exchange picture links to give participants a chance to visit the respective sites and evaluate the pictures (example 8).

(8)

20:23:31 <nice gam> pic

20:22:34 > for trade

20:22:37 <nice gam> email or link

20:22:41 > link

20:22:44 >tts? 


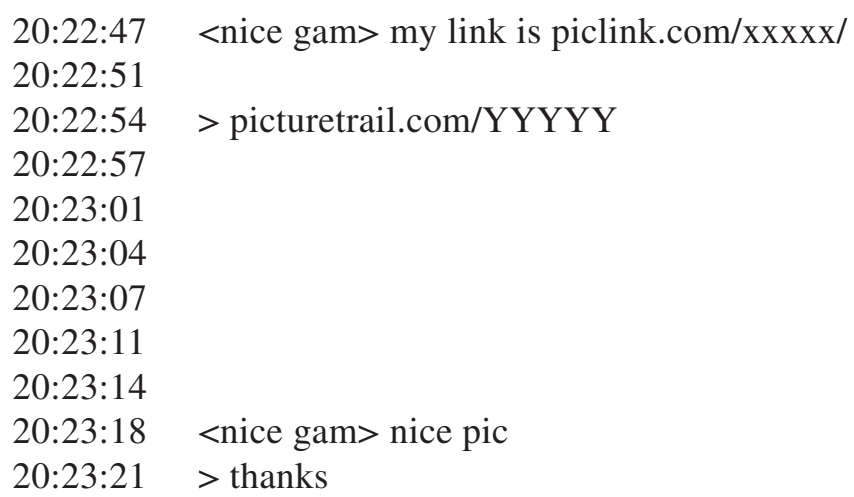

This patterning gives users a way to manage and signal the accomplishment of these different stages of the interaction. It also helps them to manage multiple interactions strategically, the breaks giving users time to engage with other interlocutors, as well as to prioritize different interactions based on the stages they have reached and the likelihood of them progressing to subsequent stages.

\section{Polychronicity and conversational synchrony}

As noted before, one thing that characterizes this activity is a high degree of polychronicity. Users often engage in conversations with multiple interlocutors in order to maximize their chances of success. These multiple, simultaneous interactions do not proceed at the same pace. Some are slower, and some are faster. Each thread of conversation follows its own rhythm, "yet [they] are perfectly coordinated with each other as they weave in and out without ever colliding" (van Leeuwen 2005: 194).

Several factors serve to facilitate these multiple concurrent interactions. First, the alternation between bursts and breaks makes it easier for users to toggle from one interaction to another. Second, interlocutors tend to synchronize their rhythm with the person they are talking to. Such "conversational synchrony" has been widely noted in face-to-face conversation (Hall 1959; Kendon 1974; Scollon 1982). Erickson (1980) has shown, for example, that in ordinary talk people speak to each other in a regular meter of regular beats and time their entrances and exits to the rhythm of these beats. As Capra (1982: 300, 302) notes, "Human communication [...] takes place to a significant extent through the synchronization and interlocking of individual rhythms [... and] opposition, antipathy, and disharmony will arise when the rhythms of two individuals are out of synchrony". This also seems to be the case in computer-mediated interaction; de Siqueira and Herring (2009), for example, in their study of dyadic IM (Instant Messaging) conversations, found that some users attempt to achieve conversational synchrony by adjusting the rhythm and timing of their contributions to fit the temporal styles of their interlocutors. 
Example 9 shows a user engaging in four different conversations at once, each conversation proceeding at a different rhythm. In this short segment, for example, the conversation with Lean Fit proceeds more quickly than that with Sporty Stylish, which proceeds more quickly than those with Gymfit, built btm, and tanguy. Each conversation, however, seems to take up its own fairly consistent pace.

(9)

\begin{tabular}{|c|c|c|c|c|}
\hline 09:26:20 & $\begin{array}{l}\text { LeanFit } \\
\text { > with family } \\
\text { >clerial }\end{array}$ & SportStylish & Gymfit built btm & $\begin{array}{l}\text { tanguy } \\
<\text { tanguy }>\text { alone? }\end{array}$ \\
\hline $09: 26: 24$ & $>\mathrm{u}$ & & & \\
\hline $09: 26: 28$ & $<$ LeanFit $>$ icic & $\begin{array}{l}>\text { what time u } \\
\text { go today }\end{array}$ & $\begin{array}{l}<\text { Gymfit built } \\
\text { btm }>\text { have icq } \\
\text { number? }\end{array}$ & \\
\hline 09:26:32 & $\begin{array}{l}<\text { LeanFit }>\text { bank } \\
\text { clerk here }\end{array}$ & & & $>$ no \\
\hline $09: 26: 36$ & $>\mathrm{ic}$ & & & \\
\hline $09: 26: 41$ & $<$ LeanFit $>$ hei & & & \\
\hline $09: 26: 45$ & $\begin{array}{l}<\text { LeanFit }>\text { u fit } \\
\text { too? }\end{array}$ & $\begin{array}{l}<\text { SportStylish }> \\
\text { what col of } \\
\text { your speedo? }\end{array}$ & $\begin{array}{l}>\text { yes, but not open } \\
\text { now }\end{array}$ & \\
\hline 09:26:49 & & $\begin{array}{l}<\text { SportStylish }> \\
\text { maybe } 1100\end{array}$ & & \\
\hline $09: 26: 53$ & $>$ medium & & & \\
\hline $09: 26: 57$ & $<$ LeanFit $>$ icic & & & \\
\hline 09:27:01 & & >arrive?? & & \\
\hline 09:27:05 & $>$ mind? & $\begin{array}{l}<\text { SportStylish }> \\
\text { around }\end{array}$ & & \\
\hline 09:27:10 & & >where u nt & & \\
\hline $09: 27: 14$ & $\begin{array}{l}<\text { LeanFit }>\text { u do } \\
\text { exercises? }\end{array}$ & & & $\begin{array}{l}<\text { tanguy }>\text { me no } \\
2\end{array}$ \\
\hline $09: 27: 18$ & $>$ no now & & & \\
\hline $09: 27: 22$ & $>$ no money & & & \\
\hline $09: 27: 26$ & & $\begin{array}{l}<\text { SportStylish }> \\
\text { hung hom. u? }\end{array}$ & & $>\mathrm{ic}$ \\
\hline
\end{tabular}

It is also important to remember that these interactions are not just coterminous but also competitive, and not all participants are equally successful in maintaining the attention of their interlocutor. The relative pace of the interactions, therefore, can be an indication of how successfully they are progressing and can also tell us something about which party is more in control of the interaction. 
In example 10, the user begins an engagement with an interlocutor called chrisboi. While he is waiting for chrisboi to respond, he answers the invitation of $A C$ Reloaded, returns to chrisboi with a reaction to his information, and then answers $A C$ reloaded's question before initiating another sequence with chrisboi.

20:19:06

20:19:09 >8“ here

20:19:13

$20: 19: 16$

$20: 19: 19$

20:19:23

$<$ chrisboi in central $>$ cool

20:19:26 <chrisboi in central $>6.5$ here $<$ AC Reloaded $>$ hello maybe

$>$ yo

$<$ AC Reloaded $>$ care for a chat?

20:19:29

$>$ sure

20:19:33

20:19:36

$>$ lol

20:19:39

$>$ sounds nice

20:19:43

$20: 19: 46$

$>$ r u gam?

$<$ AC Reloaded $>$ how's going man?

$20: 19: 50$

$<$ AC Reloaded $>$ yeah I am

$20: 19: 53$

>horny n u

20:19:56 >r u top or btm

$<$ AC Reloaded $>$ so am I

20:20:00

20:20:03

$<$ chrisboi in central $>$ am vers top

20:20:06 <chrisboi in central $>\mathrm{u}$ ?

As chrisboi begins to be more forthcoming in his responses, the user pays more attention to that conversation and less attention to AC reloaded (example 11).

20:20:10 >nice i vers

20:20:13 <chrisboi in central>ur stats btw?

$20: 20: 16$

$20: 20: 20$

20:20:23

20:20:27

$>$ gwm 28 6' $78 \mathrm{~kg} 44 \mathrm{c} 32 \mathrm{w} 8 \mathrm{cut}$

$20: 20: 30$

thick vers

20:20:33

20:20:37

$<$ AC Reloaded $>$ care to intro?

20:20:40 
20:20:43

20:20:47

20:20:50

$<$ chrisboi in central $>\operatorname{cool} \ldots$ sounds hot

$>$ gwm $286^{\prime} 78 \mathrm{~kg} 44 \mathrm{c} 32 \mathrm{w} 8 \mathrm{cut}$ $>$ do u work out

20:20:53

$<$ chrisboi in central $>$ where are $\mathrm{u}$ from?

20:20:57

20:21:00

20:21:03

20:21:07 >Melbourne

20:21:10 >u

20:21:13

20:21:17

20:21:20

20:21:24

$<$ chrisboi in central $>$ from Tokyo

20:21:34 >ok nice

20:21:37

20:21:40

20:21:44
$<$ AC Reloaded $>8$ " that's a very

size

Later, as the interaction with chrisboy heats up even more, AC reloaded gets only a minimal response ("tks" means thanks), before being completely ignored. At the end $A C$ reloaded says, "Anyway I think you must be pretty occupied at the moment, sorry for the intrusion", and terminates the interaction (example $12)$.

20:21:47 >well I dont see al that I will get

20:21:50 >lol

20:21:54 >tks

20:21:57 <chrisboi in central>lol

20:22:00

20:22:04

20:22:07

$>$ do ulive here or $\mathrm{r} u$ visiting

$<$ AC Reloaded $>$ no prob

20:22:11

20:22:14

20:22:17

20:22:21

$<$ chrisboi in central $>\mathrm{i}$ live here

$>$ same 
20:22:24 <chrisboi in central>have lived

20:22:27 here over 2 years now

20:22:31

20:22:34 >I just moved in 3 months ago

20:22:37

20:22:41

20:22:44 > wot t u into?

$<$ AC Reloaded $>$ anyway I think u must be pretty occupied at the moment, sorry for the intrusion

An important difference between these two conversations is not just timing, but the interactional roles the participants are playing. With chrisboi, the user is the one who initiates most of the sequences, asking questions, and taking rapid consecutive turns to show interest. In the other conversation, $A C$ reloaded initiates the sequences that are answered mostly with minimal responses.

\section{Timing, social identity, and power}

As can be seen from the examples above, timing and rhythm are not just a matter of conversational synchrony, but also a matter of interactional roles and social power. The way timing is managed affects and is affected by the degree of "desirability" the user is able to project through the relative "market value" of different personal and physical attributes within this community and how "desirable" he perceives his interlocutor to be. Some people, as a consequence of their perceived market value, are less willing to wait, while others who are not so desirable are forced to wait longer for responses. In fact, it is sometimes by taking longer pauses that users signal their desirability and power.

In example 13, after try has given his "stats", the user replies with his own "stats", breaking them up into three consecutive turns, not, as in the above examples, to create suspense and desirability, but rather, because the last bit of information, the user's weight, marks him as having less "market value" than his interlocutor.

20:20:06 <try> I am aged 23, $130 \mathrm{lbs}, 5 ; 11$ “ how about u?

20:20:10

20:20:13 > I am 23 also

20:20:16 >178 cm

20:20:20 >190 lbs

20:20:23

20:20:27 
20:20:30

20:20:33

20:20:37

20:20:40

20:20:43

20:20:47

20:20:50

20:20:53

20:20:57

20:21:00

20:21:03

$<$ try $>$ ic

After revealing this potentially discrediting information, he waits around $25 \mathrm{sec}$ onds before try finally replies with "ic", an ambiguous response, which sometimes signals a coming rejection. The user replies to this with the question, "do I scare you?" (example 14).

(14)

20:21:07

20:21:10 > do I scare you?

$20: 21: 13$

Three minutes and nine seconds elapse.

He then waits more than three minutes for try finally to respond again (example $15)$.

20:24:08 <try> honestly, yes but I hope you don't mind

20:24:11 > it's ok

20:24:14 > at least you are willing to tell me

$20: 24: 18<$ try $>$ I think you should join a weight loss program

20:24:21

The way this particular user described in a subsequent interview his expectations about timing contrasts sharply with the participants quoted above, who considered 10 to 15 seconds between turns reasonable. He explained:

I think I am a patient guy, I am always willing to wait. There is no time limit for me to wait. I have tried before, there is guy in the chat room talking to me, after knowing my size, he doesn't reply, and I just wait for him till I turn off the computer, but still no response. But I think it's ok, as no matter he replies or not, it won't disturb me. In fact, I have so much experience of having delays, no matter long or short. Every time, if no replying for a long time, I will type "are you here?", "busy?", “do I scare you?", but most of the time, they won't reply. 
This example reminds us that conversational style is not simply a matter of personal psychological disposition or group norms, it is also often a matter of power and ideology. Different sets of rhythmic expectations either consciously or unconsciously become submerged into the "historical bodies" (Jones 2005) of users through sustained interaction in a particular kind of social environment and in response to the values the environment reflects and the possibilities it makes available to different kinds of people.

\section{Timing and pleasure}

Finally, I would like to suggest that rhythm and timing serve not just to help users manage their conversations and judge the relative interest of their interlocutors, but that it also plays a fundamental role in the pleasure participants take in this activity.

When chatting, users not only synchronize their rhythms to the multiple rhythms of their interlocutors, but they also build up their own personal rhythms (see also de Siquera and Herring 2009), characterized by almost constant rhythmic activity. Even when they are engaged in just one or two interactions, users are rarely idle: While waiting for responses from others, they fill in the time, either chatting with someone else, scrolling though the list of names in the chat room for new possibilities, surfing the web, answering their email, or playing games. Thus, while the pace of each individual conversation might be much slower than most face-to-face encounters, this multitasking gives to this activity a constant, rapid, rhythmic character.

According to Chapple (1982), in face-to-face conversation, people associate feelings of pleasure and well-being with the rhythmicity of the interaction. Eve (2004) suggests that computer chat, with its rapid alternation of sending and receiving messages, also creates similar feelings in users. In interviews, several participants noted how "absorbing" and even "addictive" this activity of chatting with multiple prospective sexual partners could be. "Once you get into it", one said, "it can be hard to stop". Another noted, "I tend to loose track of time. Two or three hours can pass, and I don't even realize it".

Csikszentmihalyi (1982) refers to this phenomenon of becoming "lost" in an activity as "flow", a state in which a person loses "self-consciousness" and a sense of time, focusing on "the present, blocking out the past and the future" (38). Others (Trevino and Webster 1992; Webster, Trevino, and Ryan 1993) have evoked Csikszentmihalyi's theory not just to explain the pleasure people associate with CMC but also the heightened sense of presence the medium creates, despite the reduced cues it makes available. 


\section{Conclusion}

The main point of this chapter is that, despite the many extraneous factors that might affect rhythm in computer-mediated interaction, timing and rhythm can have important pragmatic functions in this medium, especially for certain communities of users engaged in focused activities, for functions such as showing involvement and interest, and for creating conversational implicature, managing and signaling different stages in the interaction, and negotiating power relations. It may also play a role in the enjoyment users experience in the activity of chatting.

I do not wish to suggest that timing is equally important or used in the same way in all CMC or even in all chat-based environments; indeed, because of its important role in communicating desire, attractiveness, and seduction, timing may take on a heightened importance in the kinds of interactions I have been describing - just as in face-to-face interaction, timing is used differently and takes on different meanings in different communicative contexts.

It is both the "bodily" nature of this communicative context that makes timing so important, and rhythm and timing that make the experience of the context more of an "embodied" one for users. Rhythm, van Leewuen (2005) points out, provides an important link between semiotic articulation and the body; it contributes not just to organizing communicative events, but also to vitalizing them, allowing, in the case of these conversations, users to reach very high levels of empathy and intimacy in a shorter time as compared to face-to-face communication. In many ways, social identities online are very much "identities of rhythm" (Capra 1982).

Future work in this area should include more studies of the timing of turn taking in computer chat integrating quantitative and statistical methods (see e.g. de Siqueira and Herring 2009; Kalman et al. 2006), as well as pragmatic and conversation analytic studies of the effects of timing on the conduct of conversation and the negotiation of meaning. It should also focus more on the specific rhythmic patterns that develop in particular genres of CMC and in particular activity types (comparing, for example, task oriented interaction with casual interaction). Finally, more work needs to be done on the psychological and relational effects of rhythmicity in computer-mediated interaction, not just in chat, but also in other types of online interaction such as multiplayer games.

Timing is an analytical site where issues of involvement, attention, pleasure, and power converge. As Prior and Shipka (2003: 230-231) argue, timing is central in all interactions for "the production of embodied chronotropes, the production of a lifeworld with a certain tone and feel, populated by a certain people and their ideas, calibrated to a certain rhythm". Different media, with the different "attention structures" (Jones 2003, 2005) they make available to users, affect the kinds of relationships and "lifeworlds" users are able to enter into and the kinds of meanings that can be made. 


\section{Note}

1. Versatile means able to participate as an active or passive partner in anal sex.

\section{References}

Barthes, Roland

1975 The Pleasure of the Text, trans. Richard Howard. New York: Hill and Wang. Bays, Hillary

1998 Framing and face in Internet exchanges: A socio-cognitive approach. Linguistik Online 1. http://www.linguistik-online.de/bays.htm

Ben-Ze'ev, Aaron

2004 Love Online: Emotions on the Internet. Cambridge, UK: Cambridge University Press.

Capra, Fritjof

1982 The Turning Point. New York: Simon and Schuster.

Chapple, Eliot D.

1982 Movement and sound: The musical language of body rhythms in interaction. In: Martha Davis (ed.), Interaction Rhythms, 28-50. New York: Human Sciences Press.

Clore, Gerald C. and Karen Gasper

2000 Feeling is believing: Some affective influences on belief. In: Nico H. Frijda, Antony S. R. Manstead, and Sacha Bem (eds.), Emotions and Beliefs: How Feelings Influence Thoughts, 10-44. Paris/Cambridge: Editions de la Maison des Sciences de l'Homme and Cambridge University Press.

Couper-Kuhlen, Elizabeth

1993 English Speech Rhythm Form and Function in Everyday Verbal Interaction. Philadelphia, PA: John Benjamins.

Couper-Kuhlen, Elizabeth and Margaret Selting (eds.)

1996 Prosody in Conversation: Interactional Studies. New York: Cambridge University Press.

Csikszentmihalyi, Mihaly

1982 Beyond Boredom and Anxiety. San Francisco, CA: Jossey and Bass.

de Siqueira, Amaury and Susan C. Herring

2009 Temporal patterns in student-advisor Instant Messaging exchanges: Individual variation and accommodation. Proceedings of the Forty-Second Hawai'i Inter-

Donath, Judith national Conference on System Sciences. Los Alamitos, CA: IEEE Press.

2004 Sociable media. In: William Sims Bainbridge (ed.), The Berkshire Encyclopaedia of Human-Computer Interaction, 631-32. Great Barrington, MA: Berkshire Publishing Group.

Erickson, Frederick

1980 Timing and context in everyday discourse: Implications for the study of referential and social meaning. Sociolinguistic Working Paper Number 67.

Austin, TX: Southwest Educational Development Laboratory. 
Eve, Robin

2004 Rhythm in interaction. Research Journal of the School of Environmental Studies, Himeji Institute of Technology 6: 191-200.

Garcia, Angela C. and Jennifer B. Jacobs

1998 The interactional organization of computer mediated communication in the college classroom. Qualitative Sociology 21(3): 299-317.

Garcia, Angela C. and Jennifer B. Jacobs

1999 The eyes of the beholder: Understanding the turn-taking system in quasi-synchronous computer-mediated communication. Research on Language and Social Interaction 32: 337-367.

Goodwin, Charles

2002 Time in action. Current Anthropology 43 (Supplement August-October 2002 Special issue "Repertoires of Timekeeping in Anthropology"): S19-S35.

Gumperz, John

1982 Discourse Strategies. Cambridge, UK: Cambridge University Press.

Hall, Edward T.

1959 The Silent Language. New York: Doubleday.

Herring, Susan C.

1999 Interactional coherence in CMC. Journal of Computer-Mediated Communication 4(4). http://jcmc.indiana.edu/vol4/issue4/herring.html

Jacobson, David

1999 Impression formation in cyberspace: Online expectations and offline experiences in text-based virtual communities. Journal of Computer Mediated Communication 5(1). http://jcmc.indiana.edu/vol5/issue1/jacobson.html

Jones, Rodney

2003 Inter-activity: How new media can help us understand old media. Paper presented at the International Conference of Historical Linguistics. Copenhagen, August 11-15.

Jones, Rodney

2005 Sites of engagement as sites of attention: Time, space and culture in electronic discourse. In: Sigrid Norris and Rodney Jones (eds.), Discourse in Action: Introducing Mediated Discourse Analysis, 144-154. London: Routledge.

Kalman, Yoram M., Gilad Ravid, Daphne. R. Raban, and Sheizaf Rafaeli

2006 Pauses and response latencies: A chronemic analysis of asynchronous CMC. Journal of Computer-Mediated Communication 12(1). http://jcmc.indiana. edu/vol12/issue $1 / \mathrm{kalman} . \mathrm{html}$

Kendon, Adam

1974 Movement coordination in social interaction. In: Shirley Weitz (ed.), Nonverbal Communication, 150-68. New York: Oxford University Press.

Lankshear, Colin and Michele Knobel

2002 Do we have your attention? New literacies, digital technologies and the education of adolescents. In: Donna E. Alverman (ed.), Adolescents and Literacies in a Digital World, 19-39. New York: Peter Lang.

Lea, Martin and Russell Spears

1995 Love at first byte? Building personal relationships over computer networks. In: Julia T. Wood and Steve Duck (eds.), Under-studied Relationships: Off the Beaten Track, 197-233. Thousand Oaks, CA: Sage. 
Lehtonen, Jaakko and Kari Sajavaara

1985 The silent Finn. In Deborah Tannen and Muriel Saville-Troike (eds.), Perspectives on Silence, 193-201. Norwood, NJ: Ablex.

Mantovani, Fabrizia

2001 Cyber-attraction: The emergence of computer-mediated communication in the development of interpersonal relationships. In: Luigi Anolli, Rita Ciceri, and Giuseppe Riva (eds.), Say Not to Say: New Perspectives on Miscommunication, 229-245. Amsterdam: IOS Press.

Markman, Kris

2004 To send or not to send: Turn construction in computer-mediated chat. Texas Linguistics Forum 48: 115-124.

Millard, William B.

1997 I flamed Freud: A case study in teletextual incendiarism. In: David Porter (ed.), Internet Culture, 145-159. New York: Routledge.

Prior, Paul and Jody Shipka

2003 Chronotopic lamination: Tracing the contours of literate activities. In: Charles Bazerman and David Russell (eds.), Writing Selves/Writing Societies: Research from Activity Perspectives, 182-238. Fort Collins, CO: The WAC Clearinghouse and Mind, Culture, and Activity.

Rintel, Sean E. and Jeffery Pittam

1997 Strangers in a strange land: Interaction management on internet relay chat. Human Communication Research 23(4): 507-534.

Rintel, Sean E., Jeffery Pittam, and Joan Mulholland

2003 Time will tell: Ambiguous non-responses on Internet relay Chat. Electronic Journal of Communication 13(1). http://www.cios.org.content.lib.utexas.edu: 2048getfile\%5CRINTEL_V13N1

Ruesch, Jurgen and Gregory Bateson

1951 Communication: The Social Matrix of Psychiatry. New York: Norton.

Schegloff, Emmanuel A. and Harvey Sacks

1973 Opening up closings. Semiotica 8: 289-327.

Shankar, Tara. R., Max VanKleek, Antonio Vicente, and Brian K Smith

2000 Fugue: A computer mediated conversational system that supports turn negotiation. Proceedings of the Thirty-Third Hawaii International Conference on System Sciences. Los Alamitos, CA: IEEE Press.

Scollon, Ron

1982 The rhythmic integration of ordinary talk. In: Deborah Tannen (ed.), Analyzing Discourse: Text and Talk. Georgetown University Roundtable on Language and Linguistics, 335-49. Washington, DC: Georgetown University Press.

Smith, Mark, J. J. Cadiz, and Byron Burkhalter

2000 Conversation trees and threaded Chats. Computer Supported Collaborative Work 9(3): 97-105.

Tannen, Deborah

1984a Conversational Style: Analyzing Talk among Friends. Norwood, NJ: Ablex.

Tannen, Deborah

1984b The pragmatics of cross-cultural communication. Applied Linguistics 5: 189-195. 
Tannen, Deborah

1989 Talking Voices: Repetition, Dialogue, and Imagery in Conversational Discourse. Cambridge, UK: Cambridge University Press.

ten Have, Paul

2000 Computer-mediated chat: Ways of finding chat partners. M/C: A Journal of Media and Culture 3(4). http://www.api-network.com/mc/0008/partners.php

Trevino, Linda K. and Jane Webster

1992 Flow in computer-mediated communication. Communication Research 19(5): 539-573.

van Leeuwen, Theo

2005 Introducing Social Semiotics. London: Routledge.

Walther, John B.

1996 Computer mediated communication: Impersonal, interpersonal and hyperpersonal interaction. Communication Research 23(1): 3-43.

Walther, John B. and Lisa C. Tidwell

1995 Nonverbal cues in computer-mediated communication, and the effects of chronemics on relational communication. Journal of Organizational Computing 5(4): 355-378.

Webster, Jane, Linda K. Trevino, and Lisa Ryan

1993 The dimensionality and correlates of flow in human-computer interactions. Computers in Human Behavior 9: 411-426. 
020_Jones.pod 506

06-04-03 10:38:00 -mlb- mlb 